\title{
Multimodal Output Specification / Simulation Platform
}

Cyril Rousseau

\author{
Yacine Bellik \\ LIMSI-CNRS \\ Université Paris XI, B.P. 133 \\ 91403 Orsay cedex, France \\ +33(0)169858106 \\ surname.name@limsi.fr
}

Frédéric Vernier

The diversity of environments, systems and user profiles leads to a contextualisation of the interaction. Initially the interaction had to be adapted to a given application and for a specific interaction context. Nowadays, the interaction has to be adapted to different situations and to a context in constant evolution [7].

This diversity of the interaction context emphasizes the complexity of a multimodal system design. It requires the adaptation of the design process and more precisely the implementation of a new generation of user interface tools. These tools should help the designer and the system to make choices on the interaction techniques to use in a given context.

In this paper, we address this issue and introduce a design process of an output multimodal system including a simulation step. We describe a platform which has been implemented on the basis of this design process. The two main platform's tools allow respectively the outputs specification and the outputs simulation of a multimodal system. Finally, this paper concludes with an application of the platform through the outputs design on a mobile phone application.

\section{OUTPUT MULTIMODAL SYSTEM DESIGN}

In this section we will present existing software life cycle models and their application for the design of an output multimodal system.

\subsection{Software Life Cycle Models}

Existing software life cycle models are particularly numerous (waterfall model, V model, incremental model, spiral model, Unified Process model, Unified Modeling Language, etc.). Each model has its own advantages and can be applied during the design of an output multimodal system with more or less success. However, the design of such a system implies specific constraints from the output multimodality domain which might call into question the model interest.

During the outputs specification, choices have to be made in function of the interaction context. A serious reflexion has to be done on the outputs specification. This specification must be as complete and valid as possible.

Moreover, the implementation cost of an output system is also very important. That is why it is necessary to validate each choice made during the outputs specification before considering the implementation stage.

\footnotetext{
Permission to make digital or hard copies of all or part of this work for personal or classroom use is granted without fee provided that copies are not made or distributed for profit or commercial advantage and that copies bear this notice and the full citation on the first page. To copy otherwise, or republish, to post on servers or to redistribute to lists, requires prior specific permission and/or a fee.

ICMI'05, October 4-6, 2005, Trento, Italy.

Copyright 2005 ACM 1-59593-028-0/05/0010 ...\$5.00.
} 
The existing models propose several approaches to validate the outputs specification (more commonly called "outputs design"). For example, incremental model creates at first a partial system which will be extended later. V model defines a validation process of each stage. Spiral model conceives a system using successive prototypes.

\subsection{Simulation Step}

We propose to reinforce the verification process with a new stage allowing the simulation of the outputs specification. This simulation is different from the test phase because it does not require the presence of end users. Thanks to this simulation, the designer will be able to notice the effects and the quality of the proposed specification. This simulation is an "application" of the specification but does not replace a prototype and a test phase. Prototypes represent a second stage in the simulation process which might be improved by the results of the first simulation.

Outputs specification is then made in an incremental way by successive prototypes. The outputs design of a multimodal system is based on a cycle model composed of three steps:

- Analysis,

- Specification,

- Simulation.

The results of the analysis step are recommendations for the next specification step. During the first iteration, these ones are extracted from the project requirements. For next iterations, the analysis carries on the simulation results of the last submitted specification.

The Figure 1 introduces a simulation step in the spiral model [5]. An iteration of the analysis and conception stages is replaced by a design cycle based on three stages: analysis, specification and simulation. The following sections present the design cycle and the three associated steps.

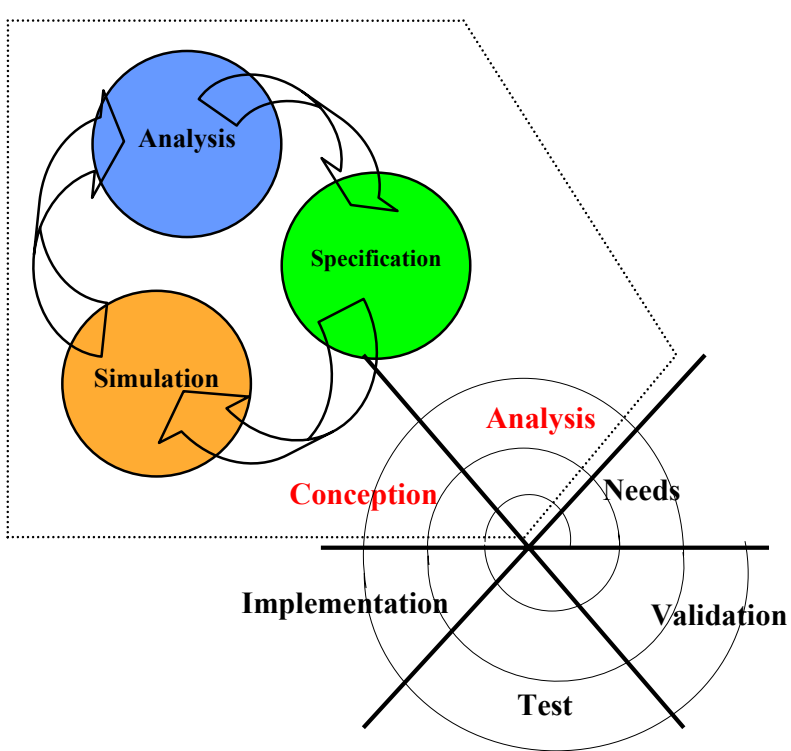

Figure 1. Simulation step in the spiral model.

\section{OUTPUTS ANALYSIS}

The analysis process consists in extracting from a data corpus, required knowledge for the outputs specification of a multimodal system. It can be divided into four tasks:

- collecting a data corpus,

- modelling the interaction context,

- identifying the interaction components,

- identifying the information units.

The Figure 2 presents the extraction process of the required elements. The following sections describe the different steps and the associated terminology.

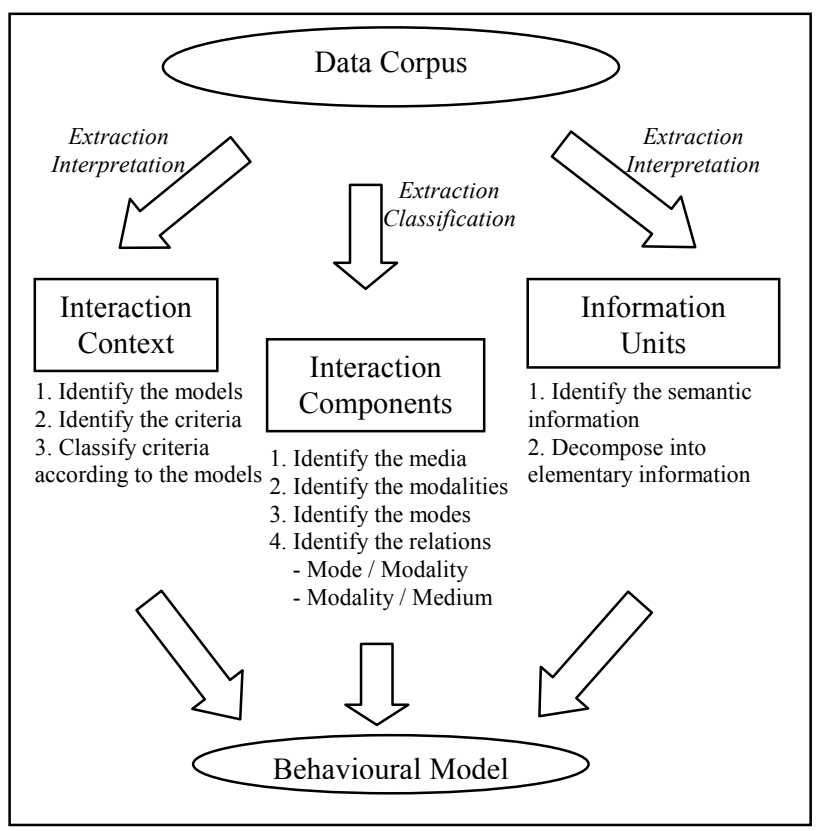

Figure 2. Extraction of the required elements.

\subsection{Requirements}

The analysis process is based on a data corpus. This corpus must be composed of scenarios / storyboards (referring to nominal or degraded situations) but also of relevant knowledge on application field, system, environment, etc. Collecting this corpus must be strictly done and should produce a consequent and diversified set of data. The corpus provides the elementary elements needed to build the output system core (the behavioural model). The quality of system outputs will highly depend on the corpus diversity.

The participation and the collaboration of three actors, is required: an ergonomist, a designer and an end user (expert in the application field). The designer and the user are mainly involved in the extraction of the elements while the ergonomist is mainly involved in the interpretation of the extracted elements. The participation of all these actors is not an essential condition. However, the absence of an actor will be probably the source of a fall in the outputs specification quality. 


\subsection{Interaction Context}

Once the corpus is collected, the interaction context must be extracted (Figure 2, left branch). According to authors, the interaction context may have different definitions. We refer to Anind Dey definition for the concept of interaction context [9]: "Context is any information that can be used to characterize the situation of an entity. An entity is a person or object that is considered relevant to the interaction between a user and an application, including the user and application themselves".

The interaction context modelling consists in identifying pertinent data which can influence the output interaction. These data are interpreted by the actors (ergonomist, designer, end user) to constitute criteria and classified by categories called models. A "model" [2] is a formalization of an entity (user, system, environment, etc.) and is composed of a set of dynamic or static criteria (device availability, user preference, noise level, etc.).

\subsection{Interaction Components}

It is now necessary to identify the interaction components which should be managed by the system (Figure 2, central branch). Three types of components are distinguished: mode, modality and medium. According to our user oriented definitions [3], output modes correspond to human sensory systems (visual, auditory, tactile, etc.). An output modality is defined by the information structure as it is perceived by the user (text, image, vibration, etc.) and not as it is represented internally by the machine. For example, if a scanned text may be represented internally by an image, but the perceived modality for the user is still text and not image. Finally an output medium is an output device allowing the expression of an output modality (screen, speaker, vibrator, etc.).

We can notice that some relations exist between these three notions. A mode can be associated with a set of modalities and each modality can be associated to a set of media. For example, the screen medium can express the text modality which is visually perceived by the user (visual mode).

Two types of relations between the interaction components are distinguished: "primary" and "secondary". A primary relation refers to a wanted effect whereas a secondary relation is a side effect. For instance, the vibration of a mobile phone is used to be perceived by the user in a tactile way. This implies a primary relation between "tactile" mode and "vibration" modality. But the sound generated by the vibrations is an example of side effect. So, a secondary relation between "auditory" mode and "vibration" modality can be added. All these relations define a diagram of the interaction components managed by the output system. The definition of the interaction components diagram is generally not a difficult task. The media are often defined in technical documentations and from media it is relatively easy to identify the desired output modes and modalities.

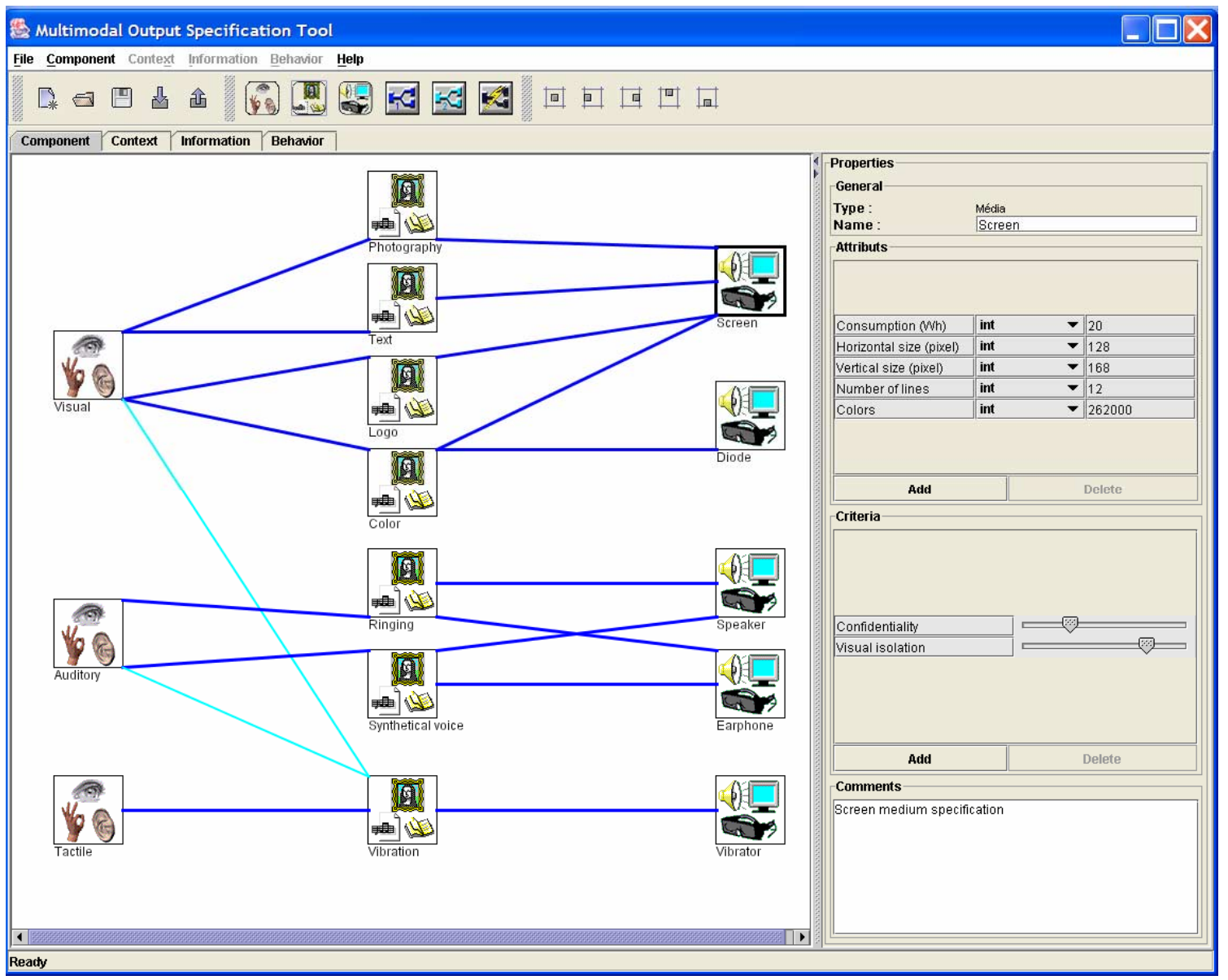

Figure 3. Edition of the interaction components managed by a mobile phone. 


\subsection{Information Units}

At last, it is necessary to identify semantic information which should be presented by the system (Figure 2, right branch). For better performance of the final multimodal system, it is recommended to decompose these informations into different semantical parts.

This problem (called semantic fission) consists in defining elementary information units from the global semantic information. For example an incoming call on a mobile phone is based on a semantic information "Call of X" which can be decomposed into two elementary information units: "the incoming call event" and "the caller identity".

\section{OUTPUTS SPECIFICATION}

The outputs specification is based on two main steps. The first step formalizes the results issued from the analysis process. This formalization allows the specification of three elements: the interaction components, the interaction context and the information units (Figure 2). During the second step these three elements are used to create the behavioural model.

\subsection{Formalization}

At first, the attributes and the criteria of each interaction component are specified. For example, the specification of a mobile phone screen (Figure 3) may result in the definition of five attributes (consumption, horizontal and vertical number of pixels, number of lines and number of colours) and two criteria (confidentiality and visual isolation). Then, the type and the possible values of each context criteria are defined. The context criterion "noise level" of the environment model can be for example an integer between 0 and 130 . In the same way the screen availability is a criterion (system model) of Boolean type. Finally, the specification of an information unit defines the domain, the criticity and its decomposition into elementary information units.

\subsection{Behavioural Model}

It is now necessary to define the behavioural model of the application. This model allows the selection of the most suitable output form to present an information unit. More precisely, it identifies the most adapted interaction components (mode, modalities and media) in regard to the current state of the interaction context. The application of the behavioural model produces an adapted multimodal presentation expressing the initial information.

A multimodal presentation is composed of a set of output (modality, medium) pairs built by redundancy or complementarity properties. For example, an incoming call on a mobile phone may be expressed through a multimodal presentation composed of two pairs. A first pair ("ringing modality", "speaker medium") indicates a phone call while a second pair ("text modality", "screen medium") presents the caller's identity.

The formalization of the behavioural model can be made in different ways: tree / graph of decision, adaptation rules [10], Petri networks, etc. Our approach uses on a behavioural model formalized by a base of election rules. This formalism has the advantage to propose a simple reasoning (If ... Then...instructions) limiting the learning cost. However this choice introduces problems on the completeness and the coherence of the rules base. Mechanisms for checking the structural coherence of the rules base have been defined but the designer is still responsible of the completeness of the rules base.

The Figure 4 presents the structure of an election rule. Three types of rules are distinguished: contextual, composition and property rules. The premises of a contextual rule describe a state of the interaction context. The conclusions define contextual weights underlining the interest of the aimed interaction components (according to the context state described in the premises rule). The composition [15] rules allow the modalities composition and so the conception of multimodal presentation with several (modality, medium) pairs based on redundancy and/or complementarity criteria [8]. Lastly, the property rules select a set of modalities using a global modality property (linguistic, analogical [4], confidential, etc.).

\begin{tabular}{|c|c|c|}
\hline \multirow{3}{*}{$\begin{array}{l}\text { Description of } \\
\text { an interaction } \\
\text { context state }\end{array}$} & Contextua & $\begin{array}{l}\text { Interest of selected } \\
\text { interaction components }\end{array}$ \\
\hline & Composition & $\begin{array}{l}\text { Complementarity and } \\
\text { redundancy properties }\end{array}$ \\
\hline & Property & $\begin{array}{l}\text { Mode, modality and } \\
\text { medium properties }\end{array}$ \\
\hline Premises & THEN & Conclusions \\
\hline
\end{tabular}

Figure 4. Structure of an election rule.

\subsection{Specification Tool}

A tool called MOSTe (Multimodal Output Specification Tool) [11] has been implemented in order to make easier the specification process. This tool is composed of four editors (component editor, context editor, information editor and behaviour editor) corresponding to each task of the specification. Contrary to existing specification tools [1], MOSTe allows the reuse of the outputs specification during the design process.

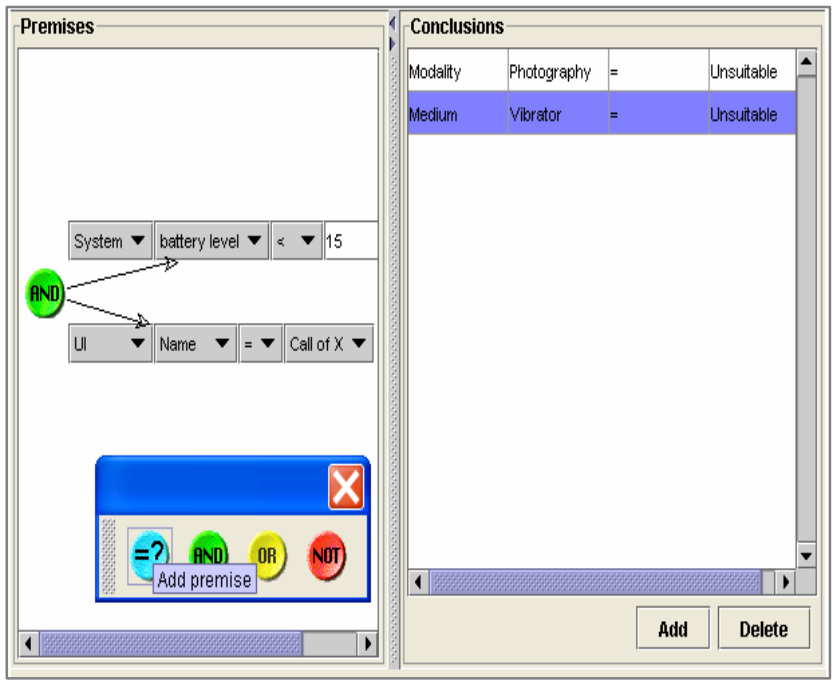

Figure 5. Edition of an election rule. 
The Figure 3 presents the interaction components editor and more precisely the edition of the interaction components managed by a mobile phone application. Concerning context and information editors, two forms allow the specification of the interaction context and of the information units. Finally, the study of an existing rule editor [14] gave us the idea to specify graphically an election rule. The specification of an election rule is based on a graph describing the rule premises and on a table presenting the rule conclusions. The Figure 5 presents a view of the behavioural model editor.

\subsection{Data Representation Language}

The resulting specification is saved in a proprietary language for futur use. This language called MOXML (Multimodal Output eXtended Markup Language), describes all the specification elements. At the present time, the definition of an outputs specification is not managed by the W3C's Extended Multimodal Annotation Markup Language (EMMA) [6]. So we defined our own data representation language based on XML with a set of tags describing all needed elements in an output multimodal system.

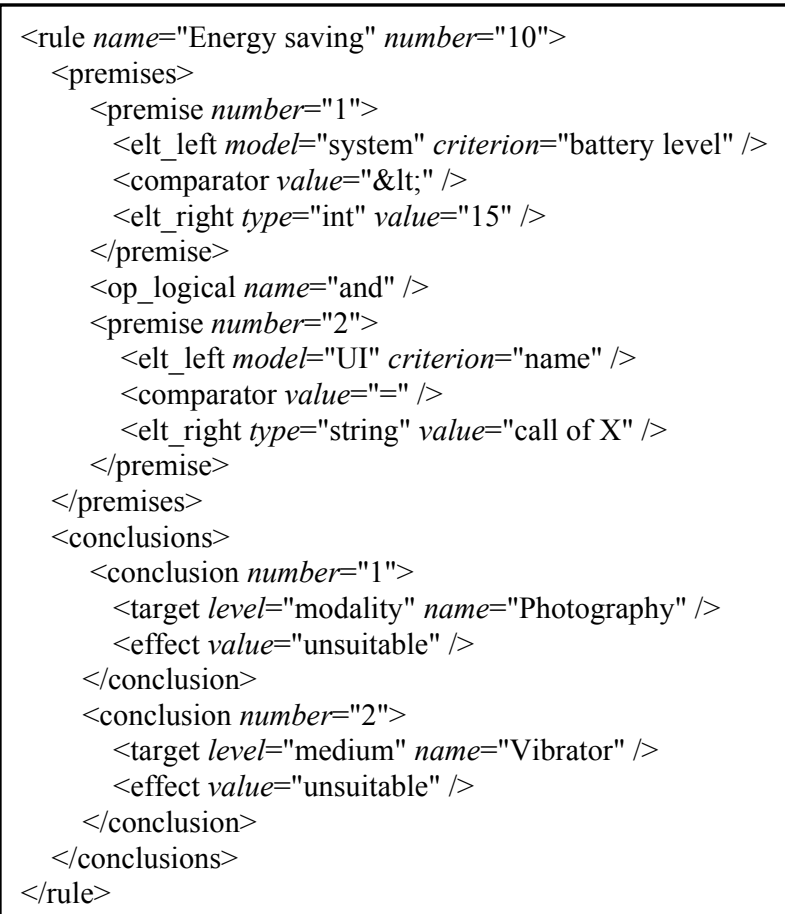

Figure 6. MOXML description of an election rule.

This Figure 6 presents the MOXML description of an election rule. This rule tries to decrease the mobile phone electric consumption to present an incoming call with a low battery level (rule premises). The proposed restrictions (rule conclusions) concern the vibrator medium and the photography modality which consumes a lot of electricity. The Figure 5 presents the graphical specification of this rule.

\section{OUTPUTS SIMULATION}

The simulation process is based on a tool allowing the execution of an outputs specification. This tool is based on a conceptual model, called WWHT and described in the following section.

\subsection{WWHT Model}

The WWHT conceptual model [13] is based on four concepts ("What", "Which", "How", "Then") describing the life cycle of an adapted multimodal presentation:

- What is the information to present?

- Which modality(ies) should we use to present this information?

- How to present the information using this(ese) modality(ies)?

- $\quad$ and Then, how to handle the evolution of the resulting presentation?

The three first concepts (What, Which and How) refer to the build process of a multimodal presentation (Figure 7). This build process can be divided into three steps.

The first step (What) called the "semantic fission" decomposes the semantic information issued from the dialog controller into elementary information. The second step (Which) allocates a multimodal presentation to express this information. For each elementary information, an "election" of the best (modality, medium) pairs according to the interaction context state is done. All these elements define a multimodal presentation expressing the initial information. The last step (How) instantiates the elected multimodal presentation. The "instantiation" process selects concrete content to express through the selected modalities and sets presentation attributes (modalities attributes, spatial and temporal parameters, etc.). Finally the "rendering engine" presents the multimodal presentation to the user.

Coherence problems can be thrown during the build process (Figure 7, "Presentation not instantiable" and "Information not presentable"). Each step is then able to call into question the results of the previous steps.

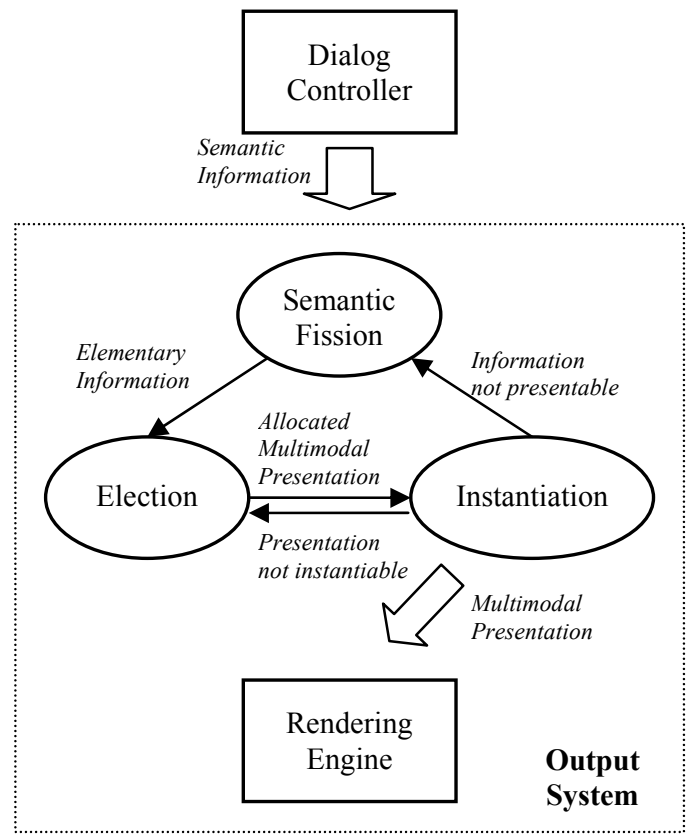

Figure 7. Presentation of a semantic information. 
The last concept (Then) is about the presentation evolution. The built multimodal presentation is adapted at the moment of it's building but it may not be the case after a context evolution. To guarantee the validity of the presentation, this one must evolve in function of the context evolution.

\subsection{Architecture Model}

The Figure 8 presents the architecture model of our simulation tool [12]. This architecture model partially applies the WWHT conceptual model. "What", "Which" and "Then" concepts are actually managed by the architecture model. Concerning the "How" concept, the implementation of a generic instantiation engine is in progress and will allow us to apply the conceptual model in full.

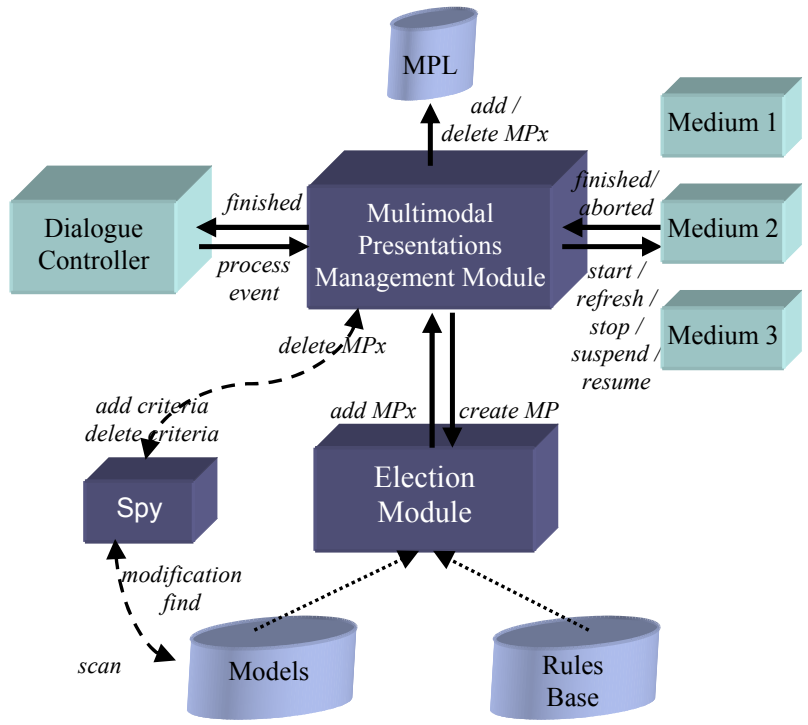

Figure 8. Software architecture.

The architecture model is composed of three main modules: election module, multimodal presentations management module and spy module. Three different structures: models (interaction context), rules base (application behaviour) and MPL (Multimodal Presentations List) define the knowledge of the system.

From the specified behavioural model, the election module allocates multimodal presentations adapted to the current state of the interaction context. The instantiation of the elected presentation is actually managed by the rendering engine (not presented in the Figure 8). Finally, the multimodal presentations management module checks the validity of persistent presentations. It receives information from the spy module which analyses the interaction context evolution.

\subsection{Simulation Tool}

A simulation tool called MOST (Multimodal Output Simulation Tool) and based on this architecture model has been implemented. This simulation tool is composed of four interfaces (Figure 9). The first interface (Figure 9, A) simulates the dialog controller. More precisely it allows the launch of the presentation process for specified information units. The second interface (Figure 9, B) simulates a context server allowing the instantly modification of the interaction context state. The third interface $($ Figure 9, C) is a system window describing the simulation results in a textual form. The last interface (Figure 9, D) presents with graphics and sounds the simulation results. Contrary to other interfaces, this interface must be specially implemented and added to the simulation tool.

This tool can also be used to develop a prototype of the complete system. The links with the other application modules (dialog controller, context module and system media) are managed by RMI (Remote Method Invocation) connections which allows to support distributed architecture. Any modification on the specification only needs a re-initialization of the simulation tool.

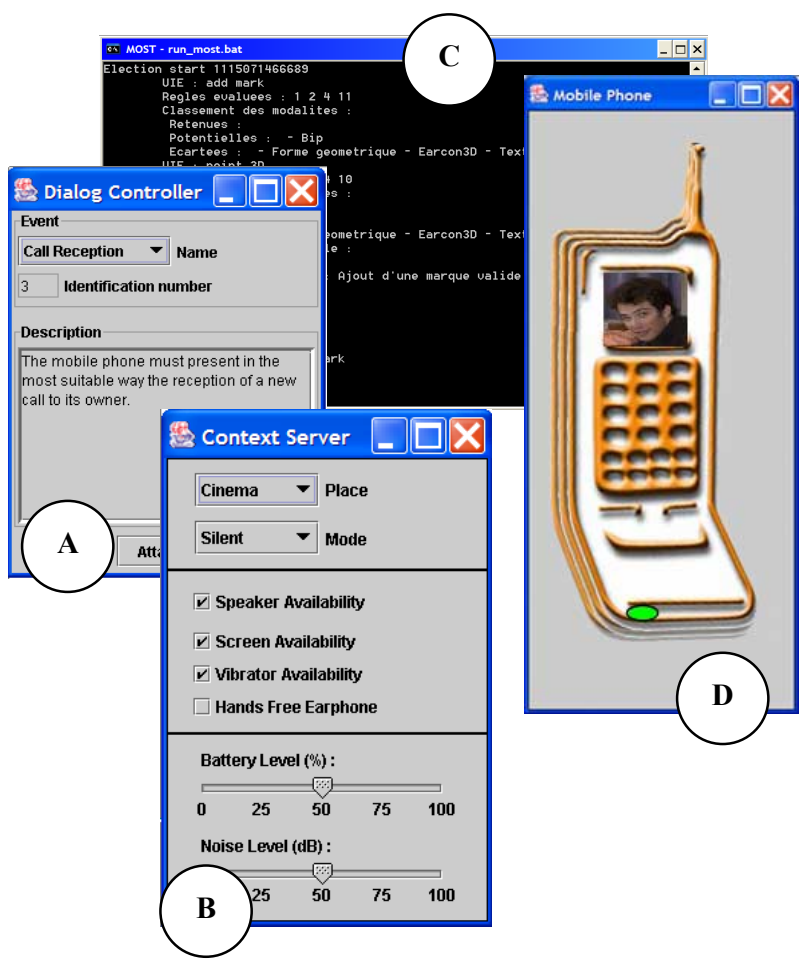

Figure 9. Simulation of an incoming call presentation on a mobile phone.

\section{APPLICATION}

The platform is currently applied in three applications:

- A first application on the mobile telephony field has been built in order to validate the election process. It aims at the simulation of an incoming call on an "intelligent" mobile phone. The call is presented in a dynamic and contextual way.

- A more complex application on the military avionics field of the INTUITION project is in integration step [13]. A first prototype has been implemented to check real time constraints and communications between the different partner's modules. This prototype is about a task of marking out a target on the ground in a fighter plane cockpit. The presentation instantiation is handled by a rendering engine specialized in avionics applications of our industrial partner (Thales-Avionics). 
- The last application concerns an air traffic control system. It will allow us to check the approach genericity and the design/specification rapidity. The implementation of a generic instantiation engine is in progress.

In the following sections, we will illustrate the application of the platform through the outputs design of the mobile phone application. More precisely, we will describe the outputs specification to present an incoming call on an "intelligent" mobile phone and two examples of specification extension.

\subsection{Incoming call on a mobile phone}

Outputs specification of this first task is presented below. The Figure 10 presents the interaction components diagram (three modes, five modalities and three media). The Table 1 modelizes the interaction context through three models (user, system and environment) and nine criteria.

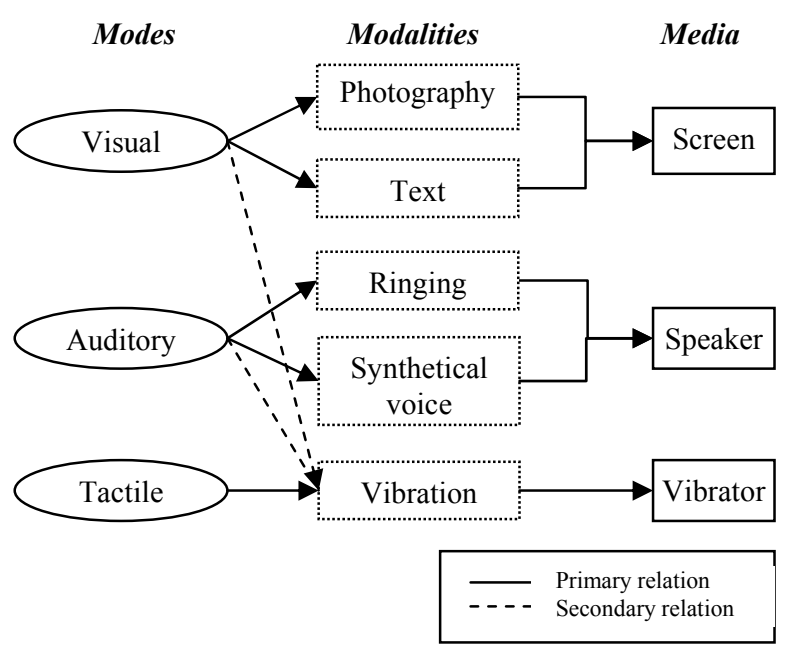

Figure 10. Interaction components diagram.

Table 1. Interaction context.

\begin{tabular}{|c|c|c|}
\hline Criteria & Values & Model \\
\hline Deaf person & Yes, No & User \\
\hline $\begin{array}{c}\text { Visually impaired } \\
\text { person }\end{array}$ & Yes, No & User \\
\hline Phone mode & Increased, Normal, Silent & System \\
\hline Screen availability & Available, Unavailable & System \\
\hline Speaker availability & Available, Unavailable & System \\
\hline Vibrator availability & Available, Unavailable & System \\
\hline $\begin{array}{c}\text { Audio channel } \\
\text { availability }\end{array}$ & Free, Occupied & System \\
\hline Battery level & $0-100$ & System \\
\hline Noise level & $0-130$ & Environment \\
\hline
\end{tabular}

Lastly, the Table 2 presents ten rules of the behavioural model: eight of contextual type, one of composition type (R5) and one of property type (R2).
Table 2. Ten rules of the behavioural model.

\begin{tabular}{|l|l|}
\hline Id & Description in natural language \\
\hline R1 & $\begin{array}{l}\text { If current elementary information is a call event } \\
\text { Then try to express it with Ringing modality }\end{array}$ \\
\hline R2 & $\begin{array}{l}\text { If current elementary information is a caller identity } \\
\text { Then try to express it with Analog modalities }\end{array}$ \\
\hline R3 & $\begin{array}{l}\text { If user is a deaf person } \\
\text { Then do not use Auditory mode }\end{array}$ \\
\hline R4 & $\begin{array}{l}\text { If user is a visually impaired person } \\
\text { Then do not use Visual mode }\end{array}$ \\
\hline R5 & $\begin{array}{l}\text { If } \text { mobile phone is in increased mode } \\
\text { Then use Redundancy property }\end{array}$ \\
\hline R6 & $\begin{array}{l}\text { If } \text { noise level is superior to } 80 \text { dB } \\
\text { Or mobile phone is in silent mode } \\
\text { Then Auditory mode is unsuitable }\end{array}$ \\
\hline R7 & $\begin{array}{l}\text { If } \text { screen is unavailable } \\
\text { Then do not use Screen medium }\end{array}$ \\
\hline R8 & $\begin{array}{l}\text { If } \text { speaker is unavailable } \\
\text { Or audio channel is already in use } \\
\text { Then do not use Speaker medium }\end{array}$ \\
\hline R9 & $\begin{array}{l}\text { If vibrator is unavailable } \\
\text { Then do not use Vibrator medium }\end{array}$ \\
\hline R10 & $\begin{array}{l}\text { If current information is a call reception } \\
\text { And battery level is low } \\
\text { Then do not use Photography modality } \\
\text { And do not use Vibrator medium }\end{array}$ \\
\hline
\end{tabular}

In a nominal situation, only $\mathrm{R} 1$ and $\mathrm{R} 2$ rules are applied. The call is then presented though a multimodal presentation composed of two pairs: (Ringing, Speaker) and (Photography, Screen). In the case of low battery level, the R10 rule changes the form of the last presentation (stops the use of the photography modality). In this case, the system adapts itself to the interaction context by choosing the Text modality to present the caller, even if it is not an analog modality (R2).

Let's suppose that during the presentation of a call reception, the noise level suddenly increases to $90 \mathrm{~dB}$. This will be at the origin of a context evolution which will lead to an invalidation of the presentation requiring a new election. The R6 rule will then change the presentation form (switch from ringing modality to vibration modality). The new presentation must be played in the last state known before the invalidation.

\subsection{Specification extension}

We want now to equip our mobile phone with a Global Positioning System (GPS) chip. The last GPS chips make possible the location of the user inside buildings. The output interaction might be adapted to the user location. To do that, a new context criterion "user location" must be added to the environment model. The list of the information units and the interaction components diagram do not need to be updated. However the behavioral model must be extended to exploit user location. For example, we can define a rule switching the mobile phone in silent phone (do not use auditory mode) when the user is in a cinema. The 
management of this new chip only needs re initialization of the simulation tool to load the updated specification.

It is also possible to extend the specification with new tasks. Let's take the example of SMS (Short Message Service) reception. This new task is similar of the first one and does not require major modifications. New rules must be defined to manage the presentation of this task according to the context. Existing rules might need modifications and more precisely the update of the rule premises to target the rule application on the first task.

\section{CONCLUSION AND FUTURE WORKS}

In this paper, we presented a platform allowing the design of an output multimodal system based on three steps: analysis, specification and simulation. This platform is composed of an analyse process to identify required elements and of two tools exploiting this analyse to specify and to simulate the system outputs. An application on the mobile telephony field has been also introduced to illustrate the platform application.

On the specification side, we are planning to add another way to formalize the behavioural model such as decision trees. In opposite of election rules, this formalism will allow a better global view of the design model. It will be possible to change the edition mode of the behavioural model from a local view (election rule) to a global view (decision tree).

Works are also in progress on the simulation tool through the implementation of an instantiation engine. This development should be made on the application of the X project concerning an air traffic control system.

Finally, our platform is actually used by our industrial partner for the design of a fighter plane cockpit simulator. An evaluation with experienced pilots is planned during the summer of this year (2005). This evaluation should help us to evaluate the platform itself.

\section{ACKNOWLEDGMENTS}

The work presented in the paper is partly funded by French DGA (General Delegation for the Armament) under contract \#00.70.624.00.470.75.96.

\section{REFERENCES}

[1] Antona, M., Savidis, A. and Stephanidis, C. MENTOR: An Interactive Design Environment for Automatic User Interface Adaptation. Technical Report 341, ICS-FORTH, Heraklion, Crete, Greece, August 2004.

[2] Arens, Y., and Hovy, E.H. The Design of a Model-Based Multimedia Interaction Manager. Artificial Intelligence Review, 9, 2-3 (1995), 167-188.

[3] Bellik, Y. Interfaces Multimodales: Concepts, Modèles et Architectures, Ph.D. Thesis, University of Paris XI, Orsay, France, 1995.
[4] Bernsen, N.O. A Reference Model for Output Information in Intelligent Multimedia Presentation Systems. In Proceedings of the ECAI'96 Workshop on Towards a standard reference model for intelligent multimedia presentation systems (Budapest, Hungary, August 1996).

[5] Boehm, B. The Spiral Model of Software Development and Enhancement. IEEE Computer, May 1988, 61-72.

[6] Chou, W., Dahl, D. A., Johnston, M., Pieraccini, R. and Raggett, D. W3C: EMMA: Extensible MultiModal Annotation markup language. Retrieved 14-12-2004, from http://www.w3.org/TR/emma/.

[7] Calvary, G., Coutaz, J., Thevenin, D., Limbourg, Q., Bouillon, L., and Vanderdonckt, J. A unifying reference framework for multi-target user interfaces. Interacting With Computer, 15, 3 (2003), 289-308.

[8] Coutaz, J., Nigay, L., Salber, D., Blandford, A., May, J., and Young, R.M. Four Easy Pieces for Assessing the Usability of Multimodal Interaction: the CARE Properties. In Proceedings of INTERACT'95 (Lillehammer, Norway, 1995).

[9] Dey, A. K., Salber, D. and Abowd, G.D. A conceptual framework and a toolkit for supporting the rapid prototyping of context-aware applications. Moran, T.P. and Dourish, P. (eds.), Human-Computer Interaction, 16, 2-4 (2001), 97-166.

[10] Karagiannidis, C., Koumpis A. and Stephanidis C.: Adaptation in Intelligent Multimedia Presentation Systems as a Decision Making Process. Computer Standards and Interfaces, 18, 7 (December 1997).

[11] C. Rousseau, Y. Bellik, F. Vernier and D. Bazalgette, Architecture framework for output multimodal systems design. In Proceedings of OZCHI'04 (Wollongong, Australia, November 22-24, 2004).

[12] C. Rousseau, Y. Bellik, F. Vernier and D. Bazalgette, Multimodal Output Simulation Platform for Real-Time Military Systems. In Proceedings of HCI International 2005 (Las Vegas, Nevada, USA, July 22-27, 2005).

[13] C. Rousseau, Y. Bellik and F. Vernier, WWHT: Un modèle conceptuel pour la présentation multimodale d'information. In Proceedings of 17th French-speaking conference on Human Computer Interaction (IHM 2005) (Toulouse, France, September 27-30, 2005)

[14] Stepien, B. (The Polished Group S.A.), 1998, from http://www.tpg.pl/RE/doc/html/UG-RulesEditor.html

[15] Vernier, F. and Nigay, L. A framework for the Combination and Characterization of Output Modalities. In Proceedings of 7th Internat. Workshop on Design, Specification and Verification of Interactive Systems (DSV-IS'00) (Limerick, Ireland, June 5-6, 2000). 2000, 35-50. 